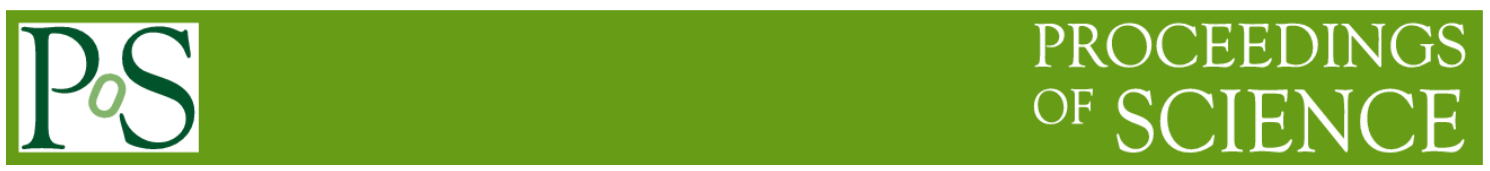

\title{
Hybrid cosmic rays detector
}

\section{Diego Andrade ${ }^{1}$}

Universidad de Guanajuato

E-mail: andradead201101icifug.ugto.mx

\section{Julián Félix}

Universidad de Guanajuato

Loma del Bosque No. 103 Col. Lomas del Campestre C.P 37150 León, Gto., México

E-mail: felix@fisica.ugto.mx

It has been developed at the Laboratorio de Partículas Elementales of the DCI, http://aboratoriodeparticulaselementales.blogspot.mx/, a four channel hybrid cosmic ray detector, that combines two detection techniques: ionization and Cherenkov light detection in a gaseous medium of $90 \% \mathrm{Ar}+10 \% \mathrm{CH} 4$. The basic detection cell consists of a quadrangular Aluminium tube of $1.01 \mathrm{~m}$ length, $2.54 \mathrm{~cm} \times 2.54 \mathrm{~cm}$ cross section, and $0.1 \mathrm{~cm}$ thickness. Furthermore, inside it has been polished to mirror. For ionization detection channels, there is a metallic Tungsten fibre coated in Gold, with diameter of one thousandth inch. The fibre is coaxially to the Aluminium tube and welded to Gold connectors which are over caps, the ones are fixed at both tube's ends. A high voltage, around2200V, is supplied to the metallic fibre which has been instrumented to read out the output signals. Besides, for Cherenkov detection channels, a Hamamatsu S10362-11-100U photodiode is placed and instrumented in each cap. Moreover the main cell, the hybrid cosmic ray detector comprises a gas system, the read out, amplification and discrimination electronic boards, as well as a data acquisition system. The DAQ system performs at $40 \mathrm{MHz}$, and writes data into a file every $1 \mathrm{~ms}$ for off line analysis. Technical information about this hybrid cosmic ray detector as the design, the construction, the characterization and the tests are treated here. The results on cosmic ray flux measurements obtained so far are discussed too.

38th International Conference on High Energy Physics

3-10 August 2016

Chicago, USA

\section{${ }^{1}$ Speaker}




\section{Introduction}

Since the discovery of the cosmic rays by the Austrian physicist Victor F. Hess in 1912[1], who demonstrated by means of a series of balloon ascents the existence of a radiation of exceptionally high penetrating capacity, several kinds of detectors have been developed. Such as the spark, cloud, or bubble chamber mainly used for particle tracking imaging; another subsequent by means of plastic scintillator or Cherenkov light detection, both complemented with photomultipliers tubes and multiwire chambers based on ionization detection. For teaching and research purposes, it has been developed a hybrid cosmic ray detector that combines both Cherenkov light and ionization detection. This is the basic cell to build a larger hybrid cosmic ray detector.

\section{Design and construction}

The detection cell is an Aluminum tube whose dimensions are, $1.01 \mathrm{~m}$ length, $54 \mathrm{~cm} \times 2.54$ $\mathrm{cm}$ cross section, and $0.1 \mathrm{~cm}$ thickness. Inside, it was polished to mirror in order to improve light reflection into the cell. There are a couple of caps for both tube's ends. Each cap is made of acrylic and painted in black to prevent the visible light pass into the cell. Figure 1 to Figure 3 illustrate the hybrid cosmic ray detector and cap designs.

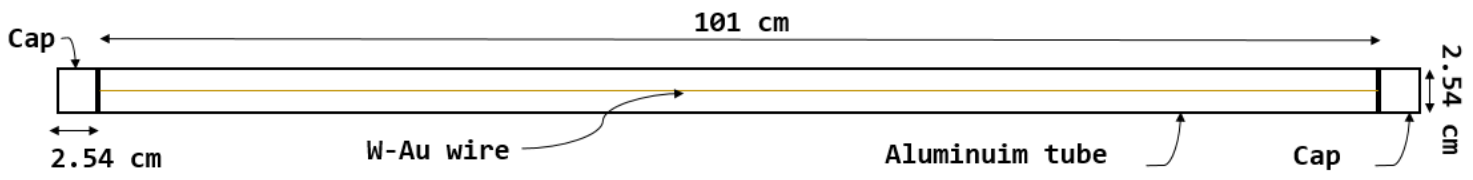

Figure 1: Cell design with dimensions,

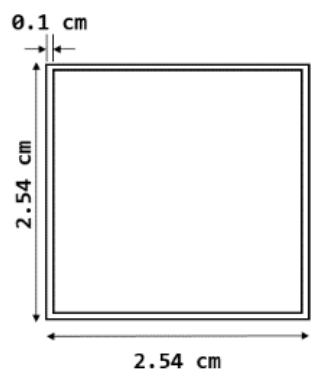

Figure 2: Transverse Aluminum tube view.
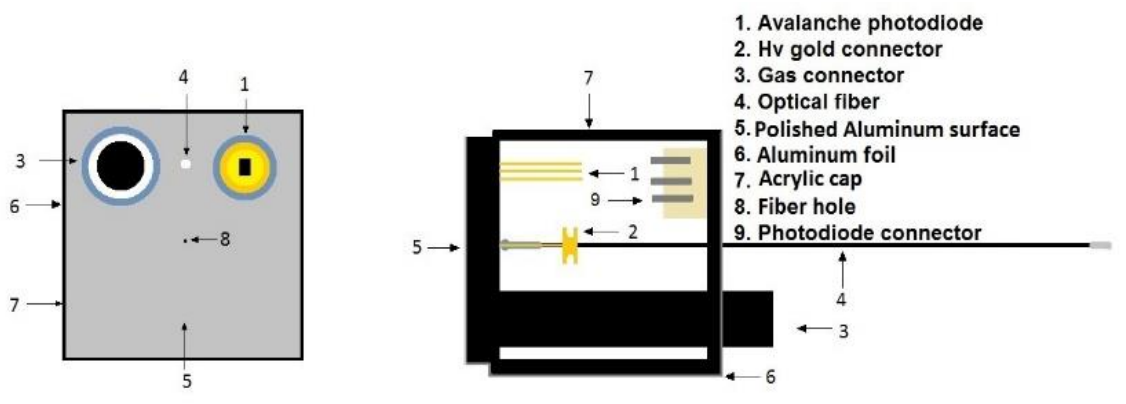

Figure 3:Cap design detail with components.

A metallic fibre made of Tungsten and coated in Gold with one thousandth inch diameter was placed coaxially to the Aluminum tube. It was welded to Gold connectors over the caps, as well as a Hamamatsu photodiode, an optical fiber, a gas connector and Aluminum foils were placed over caps. In Figure 4 are shown the assemble stages, in adittion, a detail of the cap into the cell is shown in Figure 5 and Figure 6. 


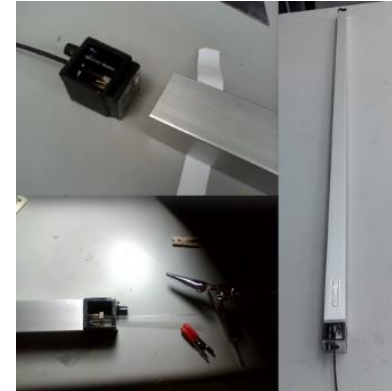

Figure 4: Hybrid detector assembling.

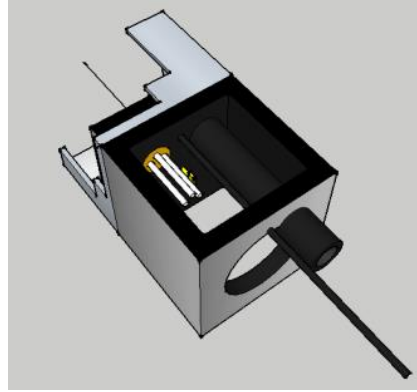

Figure 5: Detail of inserted cap into the Aluminum tube, rear perspective.

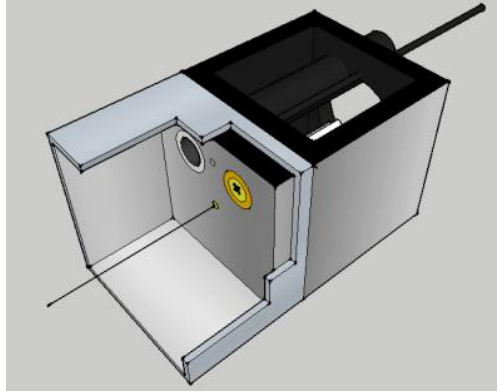

Figure 6: Detail of inserted cap into the Aluminum tube, front perspective.

Aluminium is a suitable material because of its high reflectance greater than ninety percent for the visible electromagnetic spectrum, as it is shown in Figure 7. Cherenkov light is produced close to UV region, so the photodiode S10362-11-100U used in the detector has an efficiency peak around $400 \mathrm{~nm}$ wavelength. Its detection efficiency reaches up to $65 \%$.

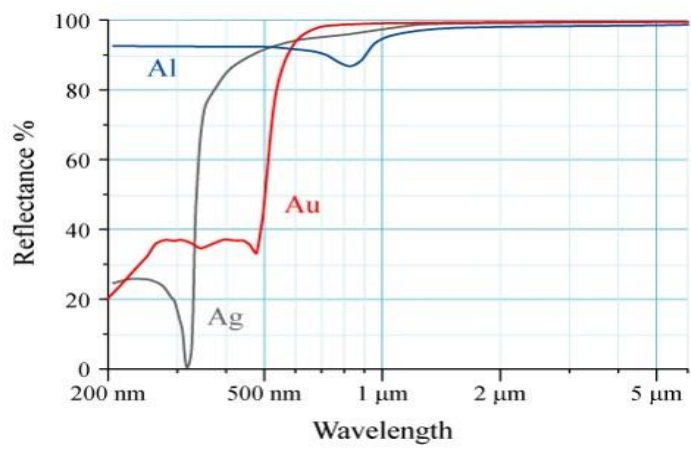

Figure 7: Reflectance for some materials[2].

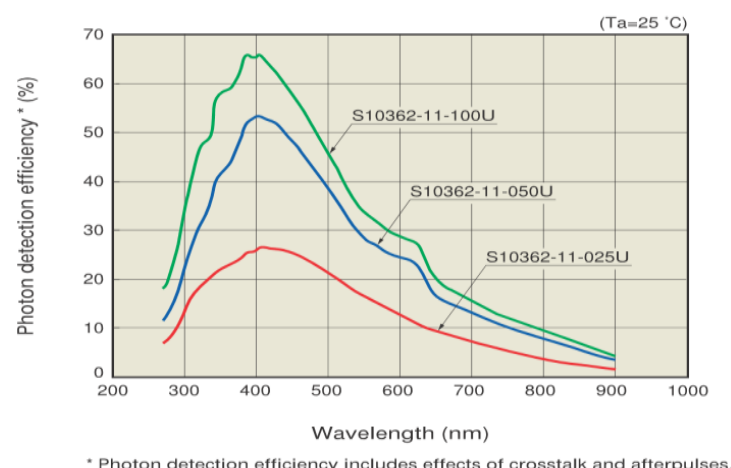

Figure 8: Photon detection efficiency vs wavelength for the photodiode Hamamatsu S10362-11100U model, green line[3].

\section{Characterization tests}

Two characterization test have been done in order to determine the suitable operation voltage and gas pressure values. Previously, the experimental system was set up as Figure 9 illustrates.
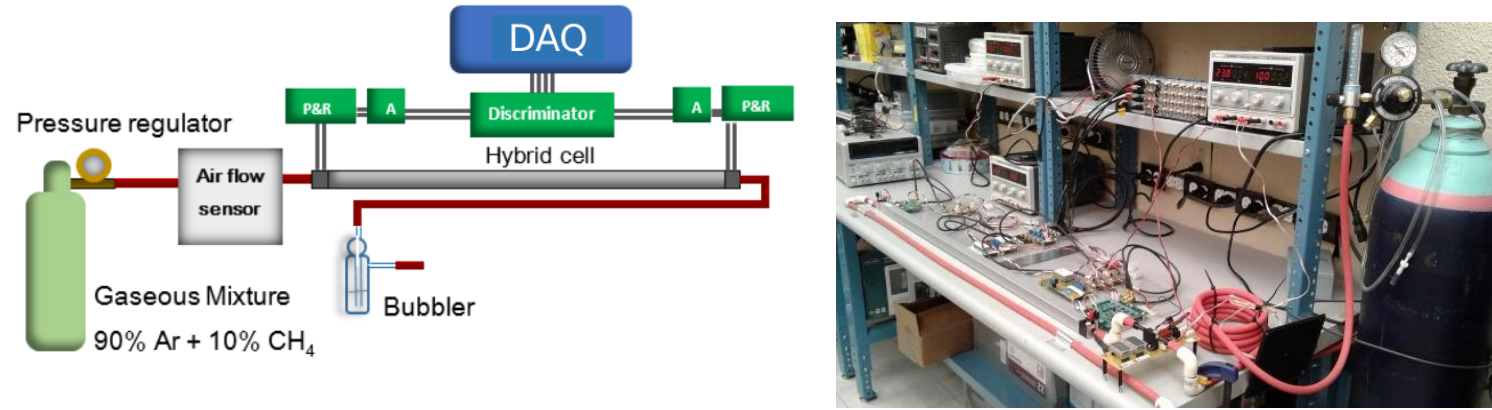

Figure 9: Experimental system connection diagram.

Figure 10: Experimental system: on the table, the hybrid cosmic rays detection cell; behind the cell, the electronic boards for power supply and read out (P\&R), amplification (A) and discrimination; beside the table, gas container and gas flow sensor attached; and on the shelf, the CompactRIO (DAQ)[4]. 

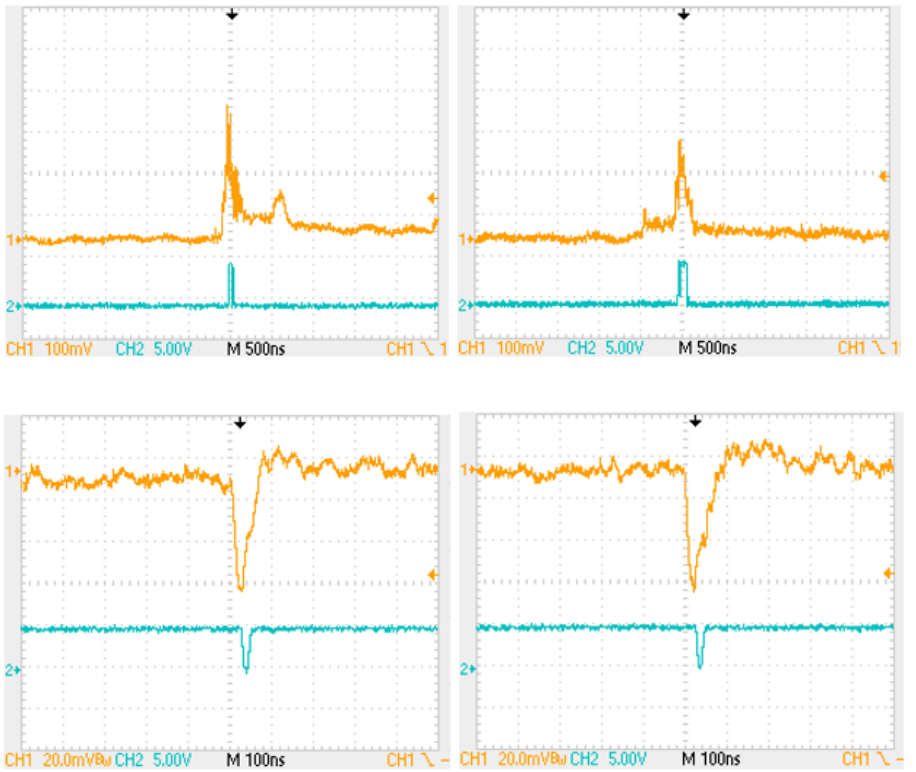

Figure 11: Typical output signals for ionization channels, on left channel 1 with $300 \mathrm{mV}$ amplitude and right channel 2 with $250 \mathrm{mV}$ amplitude. For both channels, in blue a discriminator output pulse of 5 volts amplitude and duration of $100 \mathrm{~ns}$, setting a trigger of $100 \mathrm{mV}$.

Figure 12: Typical output signals for Cherenkov channels, on left channel 1 and right channel 2 both with $60 \mathrm{mV}$ amplitude and in blue a discriminator output pulse of 5 volts amplitude and duration of $50 \mathrm{~ns}$, setting a trigger of $50 \mathrm{mV}$.

By mean of the set of codes Poisson-Superfish[5] the electric potencial inside the cell was determined as well as the electric field, by solving numerically the Laplace equation. They are shown in Figure 13 and Figure14 respectively.
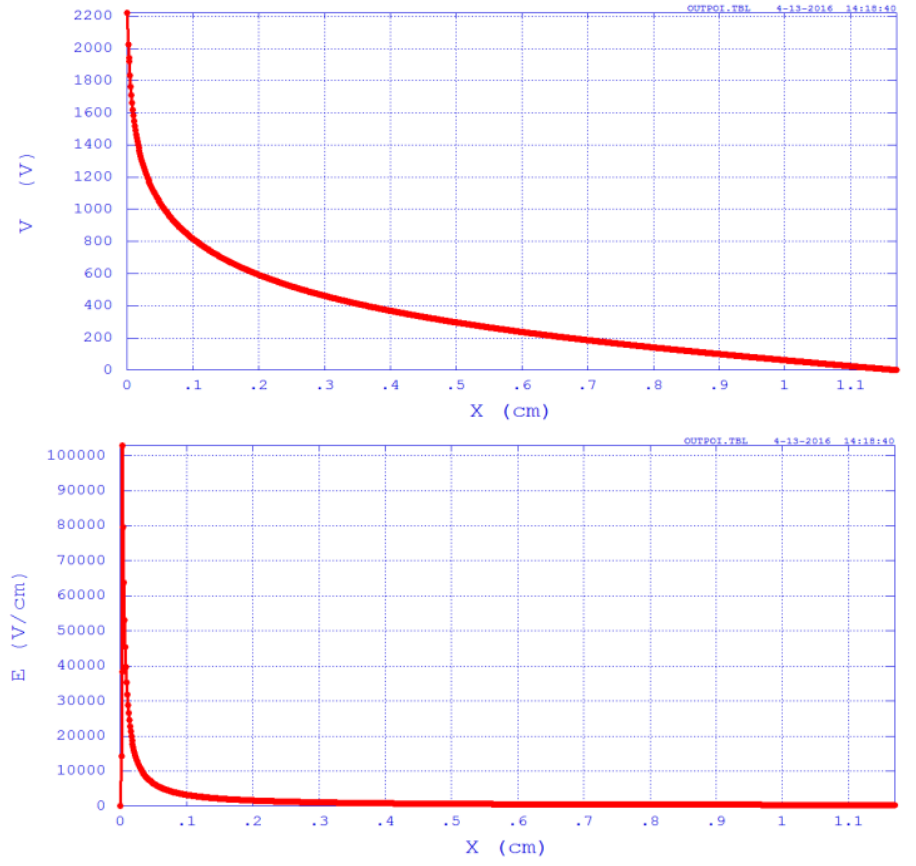

Figure 13: Graph electric potential for the hybrid cell as function of $x$ coordinate.

Figure 14: Graph $|\vec{E}|$ vs $x$ position.

\subsection{Counts as function of HV power supply}

For the ionization characterization tests, high voltage was increased in $500 \mathrm{~V}$ every five minutes starting from $500 \mathrm{~V}$ using an Ultravolt power supply which supplies up to $3000 \mathrm{~V}$. The output signals were recorded by the DAQ every $1 \mathrm{~ms}$. In similar way for Cherenkov channels, these were supplied by a BK presicion power supply from $50 \mathrm{~V}$ to $100 \mathrm{~V}$ increasing $10 \mathrm{~V}$ every 10 minutes. The suitable voltage operation found was about 2200V. Graphs for both characterization test are shown in Figures 15 and Figure 16 respectively. 

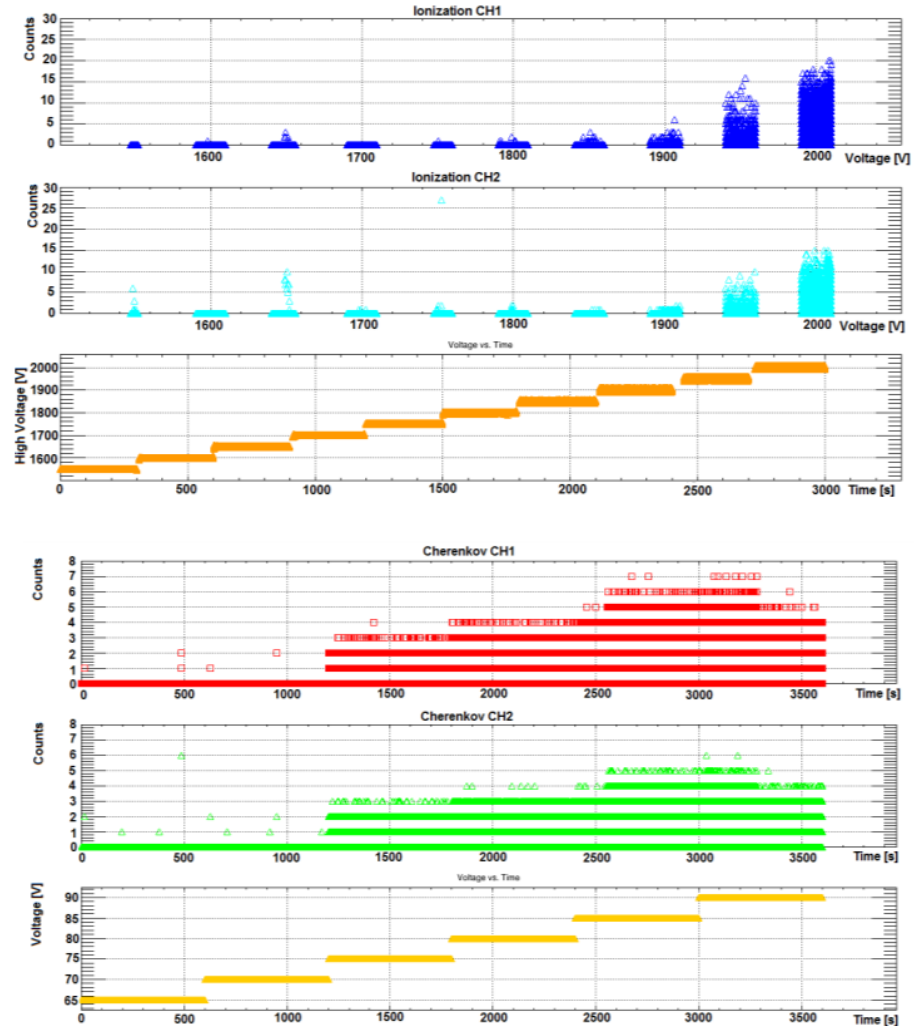

Figure 15: Characterization test, counts as function of voltage supplied. Ionization channels one (blue) and two (cyan). Vertical axis, correspond to the counts as function of the high voltage supplied in the horizontal axis.

Figure 16: Right Cherenkov channels 1 (red) and two (green). Vertical axis, correspond to the counts as function of the voltage supplied, which was increased every ten minutes (horizontal axis).

\subsection{Counts as function of differential gas pressure}

Regarding to characterization tests of counts as funtion of differencial gas pressure, this one was varied in order to determine a value that favor the detection rate. Ionization channels presented a dependence in the gas pressure. The desired value on differential pressure to operate the hybrid detector was about $0.35 \mathrm{mB}$.
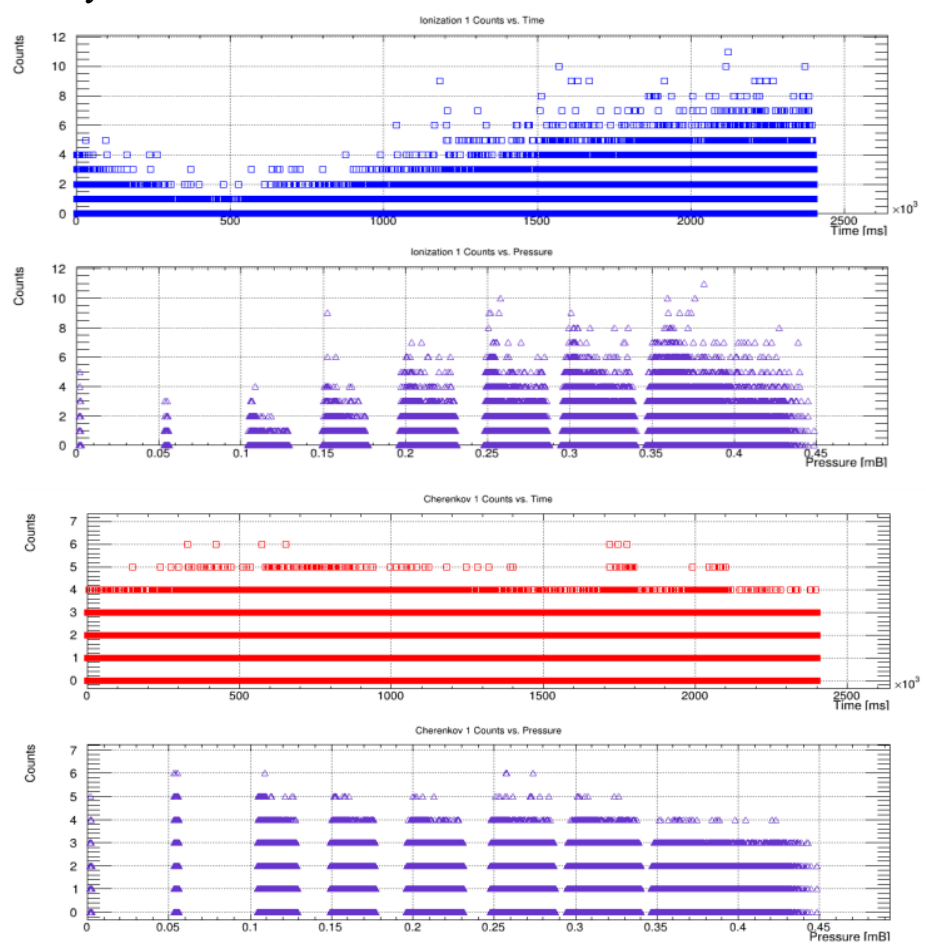

Figure 17: Characterization test, counts as function of the differential pressure for ionization channel one. Vertical axis range 0 to 12 , corresponds to the counts as function of the differential pressure, range in the horizontal axis from 0 to $0.45 \mathrm{mB}$.

Figure 18: Characterization test, counts as function of the differential pressure for Cherenkov channel one. Vertical axis range 0 to 7 , corresponds to the counts as function of the differential pressure, range in the horizontal axis from 0 to $0.45 \mathrm{mB}$. 


\section{Cosmic ray flux measurements}

In order to validate the cosmic rays counts, it has been analyzed the output signals for the ionization channel one, by means the intersection to the Cherenkov channels, that is, Ionization $\mathrm{CH} 1 \cap$ Cherenkov $\mathrm{CH} 1 \cap$ Cherenkov $\mathrm{CH} 2$. The cosmic ray flux may be measured fitting the data. Fitting the first flat region in the Figure19, the value measured for the cosmic ray flux obteined was

$\Phi(t)=p_{1} t+p_{o}$

$p_{1}=1.73 \times 10^{-7} \pm 3.28 \times 10^{-9}$

$p_{0}=5.52 \times 10^{-8}$

$\Phi(0 \rightarrow 29) \sim 15 \frac{N}{\mathrm{~m}^{2} \min }$

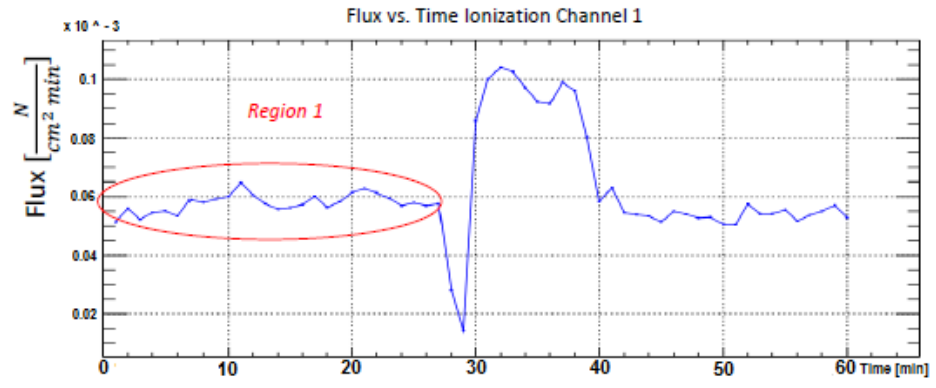

Figure 19: Cosmic rays flux measured in $\frac{N}{m^{2} \min }$ during 60 minutes

Graphs and fit parameters were calculated using Root[6].

\section{Conclusions}

We have developed a Hybrid Cosmic Ray Detector. A Hybrid Cosmic Ray Detector of two Cherenkov light and two ionization detection channels works properly. According to cosmic ray flux measurements as function of time by means the Hybrid Cosmic Ray Detector, the cosmic ray flux varies sharply and then tends to stabilize. It is not constant.

\section{Acknowledgements}

To CONACYT for the support to the project 223179, 10017, CB-2013-01 "Fondo Sectorialde Investigación para educación SEP CONACYT". To Luis Arceo, for programming the DAQ. To Víctor Armengol, Carlos Méndez, Jonathan Sánchez, Juan Segura and Miguel Vázquez, which are students from Universidad Tecnológica de la Mixteca, who designed and implemented the polishing machine for Aluminum tubes.

\section{References}

[1] N. Lectures, Physics: 1922-1941. Nobel Foundation, 1988. Singapore.

[2] M. Bass, E. W. Van Stryland, D. R. Williams, and W. L. Wolfe, Handbook of optics, vol. 2. McGraw-Hill New York, 2001. U.S.

[3] Hamamatsu, "Mppc multipixel photon counter s1036211100u datasheet." http://www.datasheetlib.com/datasheet/707356/s1036211100uhamamatsuphotonics.html, Jan2008.

[4] National Instruments, user manual and specifications NI cRIO-9025 (October, 2015). Http://www.ni.com/pdf/manuals/375490d.pdf.

[5] M. Menzel and H. K. Stokes, “Users guide for the poisson/superfish group of codes," tech. rep., Los Alamos National Lab., NM (United States), 1987.

[6] R. Brun and F. Rademakers, "Root an object oriented data analysis framework," Nuclear Instruments and Methods in Physics Research Section A: Accelerators, Spectrometers, Detectors and Associated Equipment, vol. 389, no. 1, pp. 81-86, 1997. 